\title{
Behavioral Learning of Aircraft Landing Sequencing Using a Society of Probabilistic Finite State Machines
}

\author{
Jiangjun Tang* and Hussein A. Abbass ${ }^{\dagger}$ \\ School of Engineering and Information Technology, UNSW-Canberra, Canberra, Australia \\ *Email: j.tang@adfa.edu.au \\ †Email: h.abbass@adfa.edu.au
}

\begin{abstract}
Air Traffic Control (ATC) is a complex safety critical environment. A tower controller would be making many decisions in real-time to sequence aircraft. While some optimization tools exist to help the controller in some airports, even in these situations, the real sequence of the aircraft adopted by the controller is significantly different from the one proposed by the optimization algorithm. This is due to the very dynamic nature of the environment.

The objective of this paper is to test the hypothesis that one can learn from the sequence adopted by the controller some strategies that can act as heuristics in decision support tools for aircraft sequencing. This aim is tested in this paper by attempting to learn sequences generated from a well-known sequencing method that is being used in the real world.

The approach relies on a genetic algorithm (GA) to learn these sequences using a society Probabilistic Finite-state Machines (PFSMs). Each PFSM learns a different sub-space; thus, decomposing the learning problem into a group of agents that need to work together to learn the overall problem. Three sequence metrics (Levenshtein, Hamming and Position distances) are compared as the fitness functions in GA. As the results suggest, it is possible to learn the behavior of the algorithm/heuristic that generated the original sequence from very limited information.
\end{abstract}

\section{INTRODUCTION}

To find optimal landing sequences for arrival aircraft is an NP-hard problem when the constraints of spacing between arrivals depend on aircraft types and other conditions [1]. The First-Come-First-Served (FCFS) heuristic has been used as the most common approach for sequencing aircraft [2]. FCFS simply schedules the landing aircraft based on the Estimated Time of Arrival (ETA) at the runway and the minimum separation time between two consecutive aircraft as listed in Table [1]

\begin{tabular}{|c|c|c|c|}
\hline \multirow{2}{*}{ Leading Aircraft } & \multicolumn{3}{|c|}{ Trailing Aircraft } \\
\cline { 2 - 4 } & Heavy & Large & Small \\
\hline Heavy & 96 & 157 & 196 \\
\hline Large & 60 & 69 & 131 \\
\hline Small & 60 & 69 & 82 \\
\hline \multicolumn{3}{|c}{ TABLE I } \\
\hline
\end{tabular}

MINIMUM TIME SEPARATION (IN SECONDS) BETWEEN LANDINGS AND MANDATED BY FAA [3]

FCFS schedule is easy to be implemented, and it maintains a sense of fairness. However, the landing efficiency in terms of runway throughput can't be guaranteed by FCFS when unnecessary spacing requirements exist. Therefore, many aircraft landing sequencing approaches have been proposed and automation tools have been deployed in the operational environment to increase the efficiency of the system by maximizing runway throughput while maintaining safety.

Constrain Position Shifting (CPS) [4] is a common approach in practice, which shifts an aircraft forward or backward in the FCFS schedule by a specified maximum number of positions in order to achieve a landing sequence with the smallest time span. These approaches show some advantages over FCFS, such as providing the Air Traffic Controller (ATC) with additional flexibility and decision support plots to predict landing times and positions [5]. However, they increase ATC-Pilot communication and controller workload. Therefore, a mixed approach combining FCFS and aircraft shifting methods can be a better option in the operational environment for tradingoff efficiency and ATC-pilot communications. Sequencing of aircraft landing by ATC is a more complex procedure in the real world. Many factors can come into play including weather conditions, emergency situations, and even the personality and experience of an ATC [6]. Any mistake made for aircraft landing sequencing can cause critical safety risks in aviation.

Our previous work looked at the risk assessment of aircraft landing sequencing algorithms [7]. Some critical issues were recognized by approaches based on the Computational Red Teaming (CRT) [8] concept. The previous work was not concerned with learning the behaviors that generated a sequence. CRT usually starts with no or limited knowledge about the object to be challenged at the beginning. The behavior of an object needs to be learned by CRT through observations. In order to understand aircraft landing sequencing behavior and then provide some assessments, a methodology is needed to learn and model the behavior, which can enable us to apply CRT to challenge it specifically and thus, improving it.

In this paper, we present a stochastic approach combined with a Probabilistic Finite-state Machine (PFSM) [9] and Genetic Algorithm(GA) [10] to represent and learn the behavior of aircraft landing sequencing. We developed a simulator for simulating this type of behavior, where either FCFS and CPS operate on aircraft landing sequencing in order to balance runway efficiency and ATC-pilot communications. The current simulator considers some traffic conditions including mixed 
aircraft types and their ETA sequence.

A Finite-state Machine (FSM) has been widely used as a representation in many domain of applications such as, behavioral modelling in simulating autonomous entities [11], machine learning [12], and pattern recognition [13]. It has also been applied with Evolutionary Computation for solving various problems [14] [15] [16]. In our approach, PFSM is used to simulate the behavior for sequencing landing aircraft and then GA improve it by evolving the transition probabilities for generating aircraft landing sequences with high similarity to the targeting landing sequencing from the behavior simulator. In our approach, the similarity is measured by three sequence metrics: Levenshtein [17], Hamming [18] and Position based distances. GA is applied to each of them to evolve PFSM. A comparison among the metrics is conducted for finding a better measurement.

This paper is organized as follows. The behavior simulator and problem definition of aircraft landing sequencing are described in Section III. This is followed by the proposed methodology and the three metrics for learning and modelling the behavior. Finally, the experimental results are presented for both training and testing.

\section{Problem DEFinition AND AircRAFt LANDing SEQUENCING SIMULATOR}

Given aircraft arrival sequence $A\left(a_{1}, a_{0}, \ldots, a_{n}\right)$ and their corresponding wake turbulence $W\left(w_{1}, w_{2}, \ldots, w_{n}\right)$, aircraft landing sequence $A^{\prime}\left(a_{1}^{\prime}, a_{0}^{\prime}, \ldots, a_{n}^{\prime}\right)$ can be scheduled by some approach in order to maximize/minimize certain objectives, e.g. increasing runway throughput and maintaining safety. The wake turbulence of aircraft is classified into three catalogs: Heavy $(H)$, Light $(L)$, and Small $(S)$. The minimum separation is defined in Table I and is required to constrain the inter-landing time of two subsequent aircraft.

According to the minimum separation requirements and given sequences $(A)$ and wake turbulence $(W)$, CPS can search for and construct a sequence with the minimum time span for the landing of all aircraft. Sometimes the re-scheduled aircraft landing sequence $\left(A^{\prime}\right)$ is not necessarily better than the FCFS sequence $(A)$ [7]. The data used in the simulator represent peak time data; that is, the intervals between every two aircraft estimated time of arrival (ETA) is always less than 1 minute. This inter-arrival time constraints the schedule because it is mostly less than the minimum separation requirement. 1-CPS is used in the simulator, which means aircraft position can be shifted forward or backward by 1 position only. If a time span of an aircraft landing sequence $\left(A^{\prime}\right)$ from CPS is better than the FCFS sequence $(A)$ by a certain level, the CPS sequence $\left(A^{\prime}\right)$ is used otherwise the FCFS sequence $(A)$ is used.

In summary, the aircraft landing sequencing simulator takes a number of aircraft arrival sequence $\left(A_{1}, A_{2}, \ldots, A_{m}\right)$ with associated wake turbulence $\left(W_{1}, W_{2}, \ldots, W_{m}\right)$ and generates a number of new aircraft landing sequencing $\left(A_{1}^{\prime}, A_{2}^{\prime}, \ldots, A_{m}^{\prime}\right)$ using a combination of CPS and FCFS actions in order to balance efficiency and ATC-pilot communications.
The objective of this paper is to present an approach for learning and modelling the behavior of this simulator using a Probabilistic Finite-state Machine (PFSM) and Genetic algorithm (GA). The methodology assumes knowledge of the following information:

- the original arrival aircraft sequences $\left(A_{1}, A_{2}, \ldots, A_{m}\right)$ and their corresponding wake turbulence $\left(W_{1}, W_{2}, \ldots, W_{m}\right)$, and

- a new sub-sequences generated by either CPS or FCFS. These optimized sub-sequences are used to train the algorithm. (training set).

However, the algorithm doesn't know any of the following information:

- The intents of the behavior; that is, whether the objective is to minimize or maximize the time span for landing all arrival aircraft,

- the underlying mechanism/algorithm being used for sequencing aircraft landing,

- the threshold that was used to select either CPS or FCFS, and

- the minimum separation requirements for different wake turbulence.

\section{Methodology}

\section{A. Probabilistic Finite-state Machine}

A Probabilistic Finite-state Machine (PFSM) can be defined as a tuple $A=\left(Q_{A}, \sum, \delta_{A}, I_{A}, F_{A}, P_{A}\right)$ [13], where

- $Q_{A}$ is a finite set of states;

- $\sum$ is a finite input alphabet;

- $\delta_{A} \subseteq Q_{A} \times \sum \times Q_{A}$ is a set of transition;

- $I_{A}: Q_{A} \rightarrow \mathbb{R}^{+}$is the initial-state probabilities;

- $P_{A}: \delta_{A} \rightarrow \mathbb{R}^{+}$is the transition probabilities;

- $F_{A}: Q_{A} \rightarrow \mathbb{R}^{+}$is a set of acceptable states.

$I_{A}, P_{A}$ and $F_{A}$ are functions as below:

$$
\sum_{q \in Q_{A}} I_{A}(q)=1
$$

and

$$
\forall q \in Q_{A}, F_{A}(q)+\sum_{a \in \sum, q^{\prime} \in Q_{A}} P_{A}\left(q, a, q^{\prime}\right)=1
$$

Our approach manipulates the aircraft landing sequence according to only two known inputs: $\left(A_{1}, A_{2}, \ldots, A_{m}\right)$ and their wake turbulence $\left(W_{1}, W_{2}, \ldots, W_{m}\right)$. Here, the sequence of mixed wake turbulence is the only meaningful information of traffic conditions. In order to utilize it, a PFSM can be constructed by taking each combination of wake turbulence as a state $(Q)$, whose immediate next states are all permutations of this wake turbulence combination. The transitions $\delta$ between them are governed by a set of given probabilities $(P)$. However, the total number of states will be $3^{n}$; given that there are three wake turbulence types and $n$ aircraft in a sequence. The computation cost for evaluating and evolving such kind of PFSM increases exponentially when the number of aircraft increasing.

Instead of building a very complex PFSM containing all permutations as states, we construct a number of smaller 


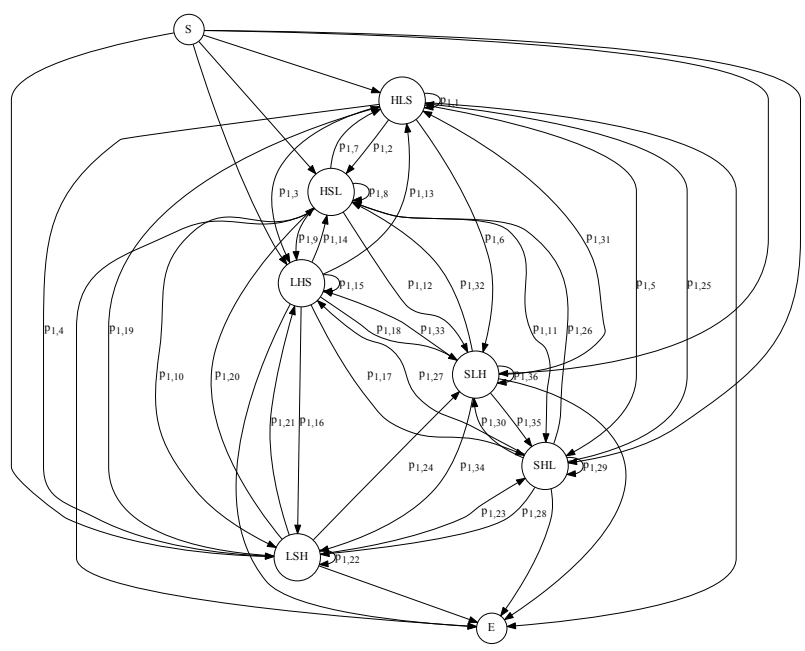

(a) PFSM1: three aircraft with three wake turbulence (H,L, and S)

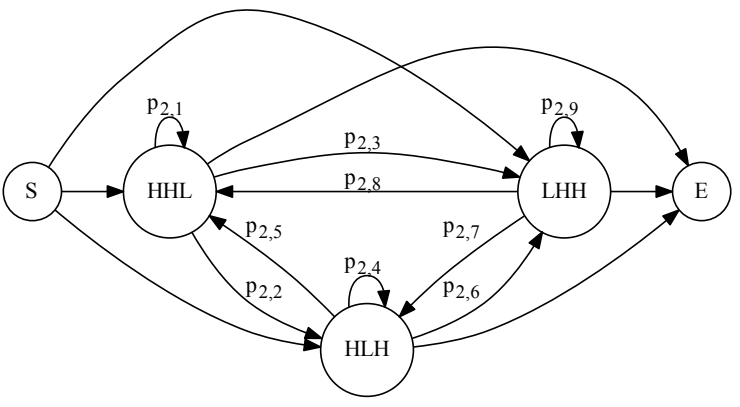

(b) PFSM2: three aircraft with two wake turbulence ( $\mathrm{H}$ and $\mathrm{L}$ )

Fig. 1. Two examples of PFSM for scheduling a sub-sequence of landing aircraft with different wake turbulence (H: Heavy, L: Large, and S: Small). $p_{i, j}$ is the transition probabilities, $i$ is the index of a PFSM, and $j$ is the index of a probability.

PFSMs. Each of them contains a group of related permutations (as states) containing the same wake turbulence types. Therefore, we decompose the aircraft sequence into a set of subsequences with length of 3 . We use this short length to capture the number of wake turbulence types. There is a total of 27 combinations that can be generated, but three of them ("HHH", "LLL", "SSS") is not necessary to be included into the PFSM. This is mainly because regardless of the sequencing algorithm being used, the wake turbulence constraint is inactive for these sequences. Hence, a total of 7 PFSMs are constructed as listed in Table III

\begin{tabular}{|l|l|r|}
\hline PFSM & States (permutations) & Number of Transitions \\
\hline PFSM1 & HLS, HSL, LHS, LSH, SHL, SLH & 36 \\
\hline PFSM2 & HHL, LHH, HLH & 9 \\
\hline PFSM3 & HHS, SHH, HSH & 9 \\
\hline PFSM4 & LLH, HLL, LHL & 9 \\
\hline PFSM5 & LLS, SLL, LSL & 9 \\
\hline PFSM6 & SSH, HSS, SHS & 9 \\
\hline PFSM7 & SSL, LSS, SLS TABLE II & 9 \\
\hline
\end{tabular}

Probabilistic Finite-STATE MACHINES BASED ON WAKE TUBURLANCE

Figure 1 depicts two examples of the PFSMs (PFSM1 and PFSM2). Figure 1(a) presents a PFSM for an aircraft sequence with three aircraft and each of them belongs to one wake turbulence. Another example of three aircraft with only two turbulence types $\mathrm{H}$ and $\mathrm{L}$ is presented in Figure 1(b). The structure of other PFSMs with only two aircraft turbulence types is similar.

As shown in the figures, There are no probabilities associated with the transitions between an initial state and its next states or between a state and the end state. In effect, this means that there are multiple initial states and multiple end states. The use of a single start and end state in the representation is mainly for convenience.
The different proposed PFSMs work together on a given arrival aircraft sequence to generate an aircraft landing sequence by Algorithm 1 . The parameter $s$ defines the number of aircraft in a temporary sequence that can be saved into the final aircraft landing sequence.

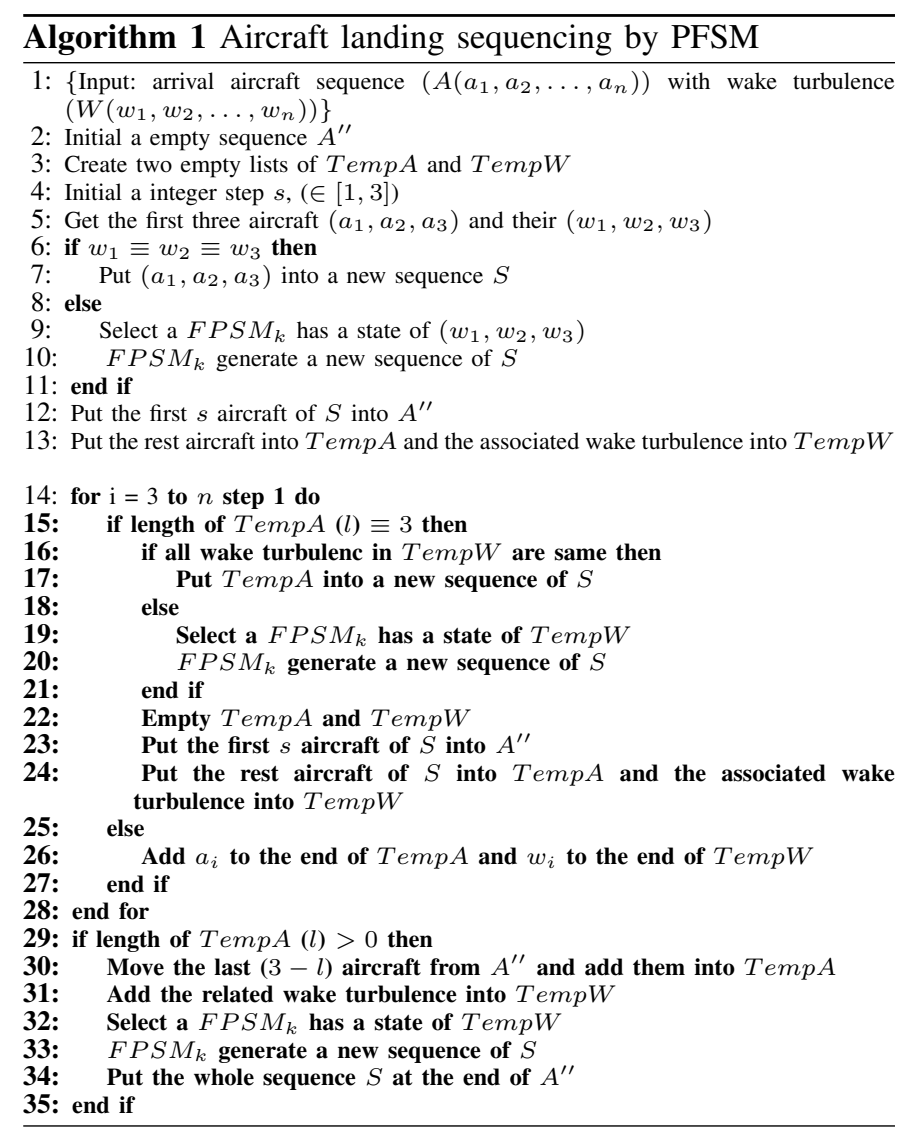


Let us take an example. Assume a sequence of "HSLH", the first three aircraft "HSL" will be input to PFSM1 and generate a new sequence. Assume that the output from PFSM1 is $S H L$ because the transition probability, $p_{1,11}$, between $H S L$ and $S H L$ is the largest transition probability in the learnt model (i.e. a maximum likelihood approach). If the step $(s)$ is defined as 1 , then the first aircraft $(S)$ is pushed into a new sequence $\left(A^{\prime \prime}\right)$, and the rest of the aircraft $H L$ and the forth aircraft $(H)$ in arrival sequence form a new temporary sub-sequence "HLH". Therefore, PFSM2 is selected. A final output is one of "HLH", "HHL", and "LHH" based on the given transition probabilities $\left(p_{2,4}, p_{2,5}\right.$, and $\left.p_{2,6}\right)$. Let us suppose that state "HHL" is selected as the output of the second PFSM, and let us assume that this is the last sub-sequence, this complete sub-sequence is pushed to the end of $A^{\prime \prime}$. In this way, a new aircraft landing sequence $(S H H L)$ is constructed.

As demonstrated by the example, the proposed PFSMs and algorithm is capable of re-constructing aircraft landing sequencing based only on the arrival sequence of aircraft and their wake turbulence without any knowledge of the actual decision module or algorithm being used to generate such sequence.

Since this is a probabilistic approach, it is necessary to develop an algorithm to learn the probabilities and as such, guides the PFMS to learn the real decision module that was used to generate that sequence. Before we discuss this algorithm, we need to discuss how two sequences are compared to calculate a measure of merit or a similarity measure.

\section{B. Sequence Metrics}

As the aircraft sequence can be transferred into a string sequence of aircraft types, string metrics are a suitable measurement to evaluate the performance of the proposed PFSM when learning and modelling of arrival aircraft sequencing behavior. Here, we use three string metrics with different biases as described below.

1) Levenshtein Distance: Levenshtein distance [17] measures the difference between two strings by the minimum number of single character edits including insertions, deletions or substitutions required to change one string into another. For two given aircraft sequences "SLLHLH" and "LLHLHS", the Levenshtein distance of them is 2, which includes deleting the first ' $L$ ' and inserting an 'S' at the end of the second sequence. Therefore, the local optimal alignment between two sequences is considered in Levenshtein distance.

2) Hamming Distance: Hamming distance [18] is a widely used metric to compare two strings with equal length. In our case, both original arrival sequence and shifted sequence have equal length, therefore, it is a suitable measurement for our PFSM. The Hamming distance is the minimum substitutions required to change one string to another; in other words, it is the number of mismatched characters between two strings. It is classically used for binary domains, but it is a generic metric independent of the size of the alphabet set. For example, given two aircraft sequences of "SLLHLH" and "LLHLHS", the Hamming distance is 5 .
3) Position based Distance: The third distance used in this paper is defined as the sum of the distances between the position of an aircraft in the original position and the shifted position of the same aircraft in the shifted position. Equation 1 describes the calculation of it.

$$
\text { PosD }=\sum_{i=1}^{l}\left|P_{i}-P_{i}^{\prime}\right|
$$

where $l$ is the length of the sequence, $P_{i}$ is the original position of aircraft $i$ in the original sequence, and $P_{i}^{\prime}$ is the shifted position in the shifted sequence. For the giving example, the position distance is $10(5+1+1+1+1+1)$ for the two sequences "SLLHLH" and "LLHLHS" because the first aircraft of ' $S$ ' is shifted to the last position (i.e. distance is $5)$, then all other five aircraft are shifted to the left by a single position. It is important to emphasize that because of the redundancy in the alphabets, when a letter is checked, it will be compared to the closest position it moved to. In other words, the first "L" in the first sequence could be the first, second or fourth "L" in the second sequence. We always assume it is the closest encounter; as such, it is the first "L" in the second sequence.

The Position based Distance is the most strict metric to measure the global similarity between two sequences regardless of their local similarities. The Levenshtein Distance considers is more local than the other two. The effect of different metrics on evaluating our PFSM for learning the behavior and modelling is investigated in Section IV

\section{PFSM Evolution}

In our approach, Genetic Algorithm (GA) is used to train our proposed PFSM to learn the behavior of aircraft landing sequencing by evolving the transition probabilities.

The length of each chromosome is equal to the total number of transitions (90) in all PFSMs as described before. The locus in the chromosome is associated to a certain transition probability in a certain PFSM. Each gene is a real number with a lower boundary of 0 and an upper boundary of 100 . Each chromosome has 7 building blocks which are mapped to the proposed 7 PFSMs respectively. The length of each building block depends on the number of transitions in a PFMS and they are varied, e.g. The length of the building block for PFSM1 is 36 while the length of the building block for others is 9. Each building block has several sub-building-blocks associated with the transitions from one state to others. To decode such a chromosome into the transitions probabilities, a group of real numbers belonging to a sub-building-block is converted into transition probabilities by normalization.

For example, nine probabilities (from $p_{2,1}$ to $p_{2,9}$ ) exist in PFSM2 as shown in Figure 1(b) Therefore, there are nine loci in a chromosome associated with this PFSM and form one building block. This building block contains 3 sub-building blocks corresponding to the three groups of transition probabilities: $\left(p_{2,1}, p_{2,2}, p_{2,3}\right),\left(p_{2,4}, p_{2,5}, p_{2,6}\right)$, and $\left(p_{2,7}, p_{2,8}, p_{2,9}\right)$. According to the definition of PFSM de- 
scribed in Section III-A the genes $(g)$ on these loci are normalized into transition probabilities satisfying:

$$
\begin{aligned}
& p_{2,1}+p_{2,2}+p_{2,3}=1 \\
& p_{2,3}+p_{2,5}+p_{2,6}=1 \\
& p_{2,7}+p_{2,8}+p_{2,9}=1
\end{aligned}
$$

After determining the transition probabilities of PFSMs, an aircraft landing sequence $\left(A^{\prime \prime}\right)$ is produced by a given aircraft arrival sequence $(A)$. Then $A^{\prime \prime}$ is compared against the corresponding landing sequences $A^{\prime}$ from the aircraft landing sequencing simulator by one of the string metrics $(d)$ as explained in the last section. As our PFMS is a scholastic approach for aircraft landing sequencing, it requires multiple evaluations of each chromosome to approximate the fitness:

1) Construct a PFSM by a chromosome

2) For each pair of given $A_{i}$ and $A_{i}^{\prime}$

a) Generate an aircraft landing sequence $\left(A_{i, j}^{\prime \prime}\right)$ by PFSM with the input of $A_{i}$

b) Calculate the sequence metric: $d_{i, j}=\operatorname{Dist}\left(A_{i, j}^{\prime \prime}, A_{i}^{\prime}\right)$;

c) Repeat Step $a$ and $b$ until a number $(T)$ of evaluations researched;

d) Get the mode $\left(D_{i}\right)$ from all $d_{i, j}$;

3) Get the mode $(D)$ from all $D_{i}$ as the fitness of the chromosome.

From the above steps, the fitness of a chromosome can be defined by Equation 2 .

$$
F=\operatorname{Mode}_{i=1}^{n}\left(\operatorname{Mode}_{j=1}^{T}\left(\operatorname{Dist}\left(A_{i, j}^{\prime \prime}, A_{i}^{\prime}\right)\right)\right.
$$

Where, $i$ is the index of a pair of aircraft arrival and landing sequences in a given set, $n$ is the total number of aircraft sequences in a given set, $j$ is the index of an evaluation for a chromosome, and $T$ is the total number of evaluations on a chromosome. The function of Dist can be any one of the three sequence metrics as mentioned above.

The objective of our GA is to minimize $F$. Binary tournament selection is used to choose parents and then Uniform Crossover is applied for producing offspring. When the mutation happens, a random real number between 0 and 100 replaces the old gene. GA stops when a predefined number of generations is reached.

\section{EXPERIMENT AND RESULTS}

\section{A. Experiment Design}

A total of 200 unique aircraft landing sequences (each sequence has 20 aircraft) are generated randomly. They are all being fed into the simulator. If the savings on the time span are at least 5\% time savings, the re-scheduled sequence from CPS is saved otherwise FCFS is chosen. This is consistent with a realistic operational environment constraints, where there is a need to balance efficiency and ATC-pilot communications. The first half of each sequence (100 aircraft) is used for training, while the second half for testing.

A total of 90 transition probabilities is required to construct all 7 PFSMs as explained in Section III-A.
GA is then used to evolve transition probabilities for training the PFSM on the training set in order to learn and model the aircraft landing sequencing behavior of the simulator. The parameters used in GA is listed as follows:

- population size: 100

- number of generation: 1000

- crossover rate: 0.9

- mutation rate: $1 / l$, the reciprocal of the chromosome length $(l)$, which is 90 in this experiment.

- The initial generation initializes the chromosomes randomly from uniform distributions.

As we have three different sequence metrics for fitness calculations, we run GA on each metric using ten different seeds. The training results are presented in the next section.

After training, the best individual in the population is selected from each of three metrics respectively and is tested on the test set. The test results are provided in Section IV-C

\section{B. Training Results}

The evolutions of our PFSM from three different sequence metrics are shown in Figure 2. The first three illustrate the average fitness values along the generations for three sequence metrics respectively. Figure 2(d) is showing the comparison of different sequence metrics.

As expected, the fitness derived from Levenshtein distance has the lowest magnitude and the fitness from Position distance has the highest magnitude. The fitness from Position distance shows the largest variations between runs as shown in Figure 2(d)

Since each metric is providing different magnitudes, it can happen that the best solution found by each metric appears to be different in fitness, but it is actually the same in terms of decision variables (i.e. probabilities). Therefore, we continue the analysis by taking the best solution found by one metric in each generation and evaluate it also on the other two metrics. An example of the best solution found over all runs using Leveshtein distance is presented in Figure 3 . This solution is also being evaluated on the other two metrics in the figure.

Although the fitness is based on Levenshtein distance, the other two generally follow the same trend of the fitness function. One interesting point is that the fluctuations of Hamming distance is quite similar to Position distance, although their magnitudes are at different levels. Similar circumstances are also found when we investigated the best runs from Hamming distance or Position distance.

Since we are evaluating the same individual in the figure at each generation, the fluctuations in the Levenshtein distance is due to the stochastic nature of the solution. However, clearly there are different sources causing different types of fluctuations when this solution is evaluated on the other two metrics. To isolate the two sources of fluctuations: those because of stochastic representation and those because of the metric itself, we measured the correlation coefficient between the Levenshtein distance and other two. The correlation coefficient was 0.92 and 0.91 in relation to the Hamming and Position distances respectively. This indicates that there is a 


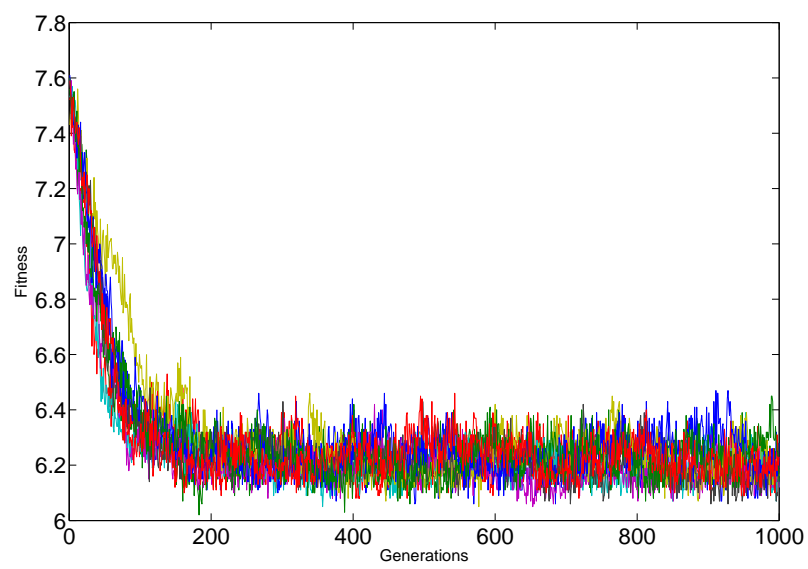

(a) Levenshtein distance

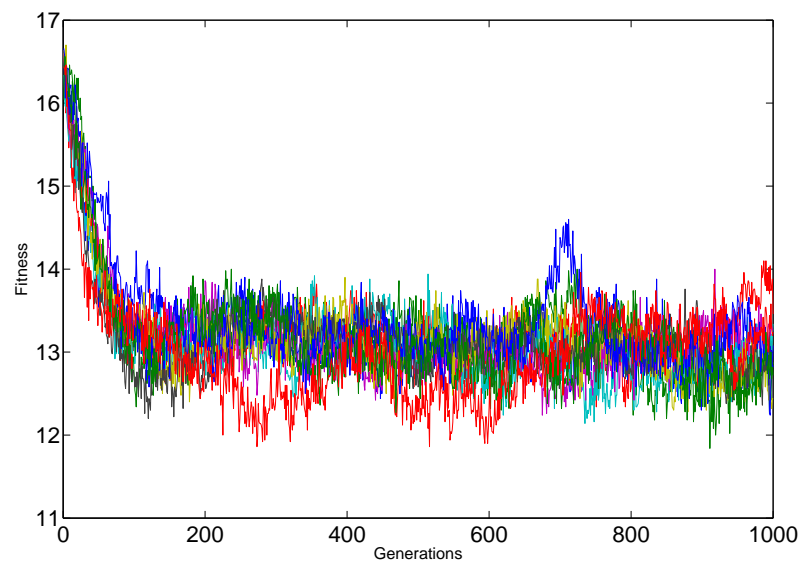

(c) Position distance

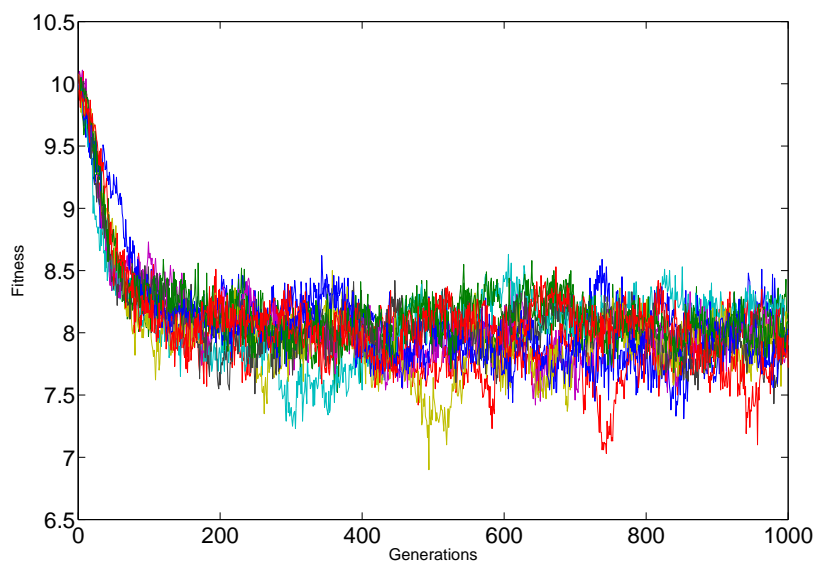

(b) Hamming distance

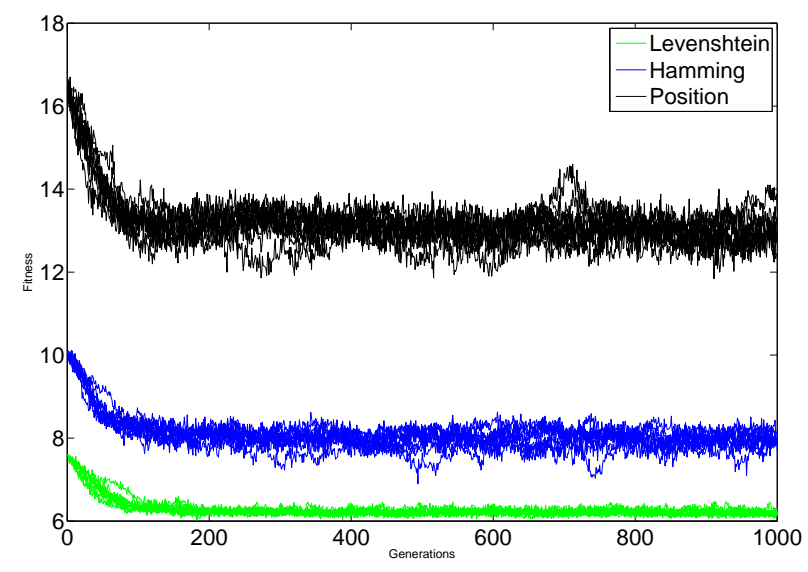

(d) Comparison of metrics

Fig. 2. The process in average fitness values for all three sequence metrics along with generations

small amount of extra fluctuations that are due to the metrics themselves.

Three best individuals in terms of their evaluated sequence metrics from the 30 runs are selected and compared in Table III

\begin{tabular}{|l|l|c|c|c|}
\hline \multirow{2}{*}{ Individuals } & \multirow{2}{*}{ Fitness function } & \multicolumn{3}{|c|}{ Sequence Metrics } \\
\cline { 3 - 5 } & & Levenshtein & Hamming & Position \\
\hline Ind_Lev & Levenshtein & $\mathbf{4}$ & 6 & 10 \\
\hline Ind_Ham & Hamming & 4 & $\mathbf{4}$ & 12 \\
\hline Ind_Pos & Position & 4 & 6 & $\mathbf{6}$ \\
\hline
\end{tabular}

THE FITNESS OF THE BEST INDIVIDUALS FOUND BY EVOLUTION USING EACH METRIC AND BEING EVALUATED ON THE OTHER TWO SEQUENCE METRICS.

The individual evaluated by Levenstein distance has a small Hamming distance but a larger the Position distance as shown in the first row in the table. The individual evaluated by Position distance is also able to produce both small Levenshein and Hamming distances as listed in the last row. However, small Hamming distance can't guarantee a small Position distance as being demonstrated by the individual evaluated by Hamming distance in the second row. These three individuals listed in the table are also used for testing our approach using the test set in the next section.

When looking at some specific aircraft landing sequencing, we find that Levenshtein distance prefers to give low error in two sequences when they have some local matching sequences. For example, "HHLSLLLSHHSLLSSSLHHL" and "HHSLLLLSSHHSLLSSLHHL", where Levenshtein distance is 4 because there are three local sequence that are matched between two sequence, which are "HH", "LLLS", and "SSLHHL". However the Hamming distance is 6. But the Position distance is 10 caused by the low global matching between them.

\section{Test Results}

The aircraft arrival sequences in the test set is input to the three individuals (PFSMs), Ind_Lev, Ind_Ham, and Ind_Pos, respectively. Each PFSM produces only one landing sequence for a given arrival sequence using a maximum likelihood 


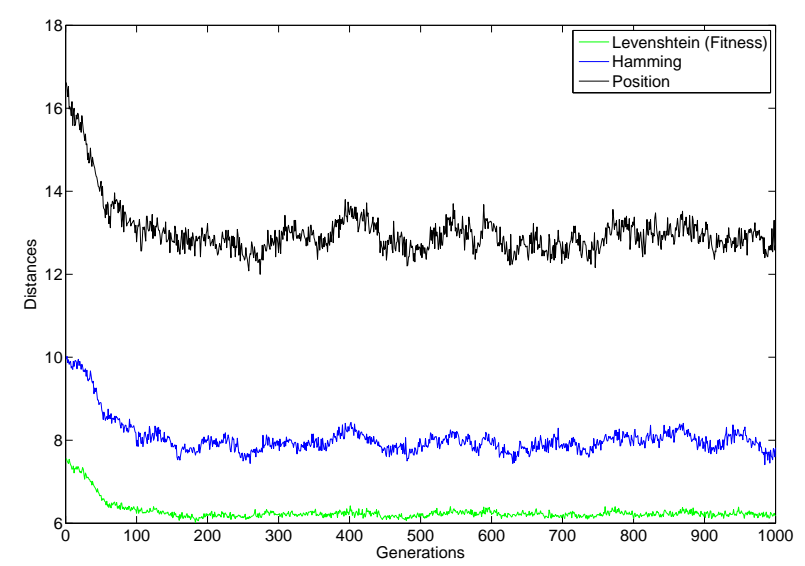

Fig. 3. The best run of fitness calculated by Levenshtein distance and evaluated also on the other two metrics measured on the same individuals

approach. The new landing sequence is compared against the sequence from the simulator. Table [IV presents the mode of the test results from three sequence metrics for all three individuals. Similar to the training session, both individuals that have been evaluated by the Hamming and Position distances can produce small Levenshtein distance.

\begin{tabular}{|l|c|c|c|}
\hline \multirow{2}{*}{ Individuals } & \multicolumn{3}{|c|}{ Sequence Metrics (mode) } \\
\cline { 2 - 4 } & Levenshtein & Hamming & Position \\
\hline Ind_Lev & $\mathbf{6}$ & 8 & 14 \\
\hline Ind_Ham & 7 & $\mathbf{6}$ & 12 \\
\hline Ind_Pos & 4 & 6 & $\mathbf{8}$ \\
\hline
\end{tabular}

THE BEST INDIVIDUALS AND THEIR TEST RESULTS ON THREE SEQUENCE METRICS

The distributions of distances on different sequence metrics between the new sequences and the target sequence for each individual are visualized as box charts in Figure 4 Overall, all the distance distributions from these three individuals are quite similar to each other in terms of means, 25 th percentiles, and 75 th percentiles. The position distances produced by the three individuals all have large variance but the variance of Levenshtein distance are always smaller than the other two. As illustrated in the chart, the individual evaluated by Hamming distance has the lowest outlier.

The number of produced sequences from each individual satisfying the following conditions are counted and listed in Table V:

- the Levenshtein distance is less than 6 representing the mode of the Levenshtein distances of Ind_Lev

- the Hamming distance is less than 6 representing the mode of the Hamming distances of Ind_Ham

- the Position distance is less than 8 representing the mode of the Position distances of Ind_Pos

As seen from the table, the individual evolved using Levenshtein distance performed worse than the other two. The individual evolved using Position distance has the best perfor-

\begin{tabular}{|l|c|c|c|}
\hline \multirow{2}{*}{ Individuals } & \multicolumn{3}{|c|}{ Number of Sequences } \\
\cline { 2 - 4 } & Levenshtein $\leq 6$ & Hamming $\leq 6$ & Position $\leq 8$ \\
\hline Ind_Lev & 56 & 20 & 20 \\
\hline Ind_Ham & 64 & 37 & 31 \\
\hline Ind_Pos & 63 & 41 & 37 \\
\hline
\end{tabular}

THE NUMBER OF SEQUENCES FROM EACH INDIVIDUAL

mance in terms of high number of sequences below the given errors for all three sequence metrics.

As demonstrated here, the PFSM approach is capable to learn and model the aircraft landing sequencing behavior and produce good matching landing sequences for the test data.

\section{CONCLUSION}

In this paper, we proposed a stochastic approach combined with PFSM and GA to learn and model aircraft landing sequencing behavior. As the experiment results suggested, this approach is capable to achieve the learning objective while only knowing limited information.

Three different sequence metrics are used for the fitness function in the GA. These metrics showed different preferences when evolving the transition probabilities of PFSM. Levenshtein distance considers the local matching sequence more than the other two, while the Position distance is more strict in terms of global matching. Therefore, the fitness values of Position distance shown in the training session are more varied than the other two. All three metrics are able to guide GA to find a set of good transition probabilities for the proposed PFSM. However, the global metrics, e.g. Position distance, has demonstrated the best results on the test set, where it produced a higher number of aircraft landing sequencing with lower errors than the other two. The results show that the proposed approach is capable of learning the underlying mechanism that generates a landing sequence.

In the future, we will introduce more uncertainty variables such as weather conditions and emergency situations to disturb the sequences during training. We will also investigate biased initialization by relying on statistical estimation methods to initialize the population in the GA instead of using a purely random initialization approach. In general, learning the mechanism that generated a solution can contribute to many subfields in evolutionary computation including surrogate models, simulation-based optimization, and fitness landscape analysis.

\section{ACKNOWLEDGEMENT}

This work has been funded by the Australian Research Council (ARC) discovery grant number, DP140102590: Challenging systems to discover vulnerabilities using computational red teaming.

\section{REFERENCES}

[1] X.-B. Hu and W.-H. Chen, "Genetic algorithm based on receding horizon control for arrival sequencing and scheduling," Engineering Applications of Artificial Intelligence, vol. 18, no. 5, pp. 633-642, 2005.

[2] A. R. Odoni, J.-M. Rousseau, and N. H. Wilson, "Models in urban and air transportation," Handbooks in operations research and management science, vol. 6, pp. 107-150, 1994. 


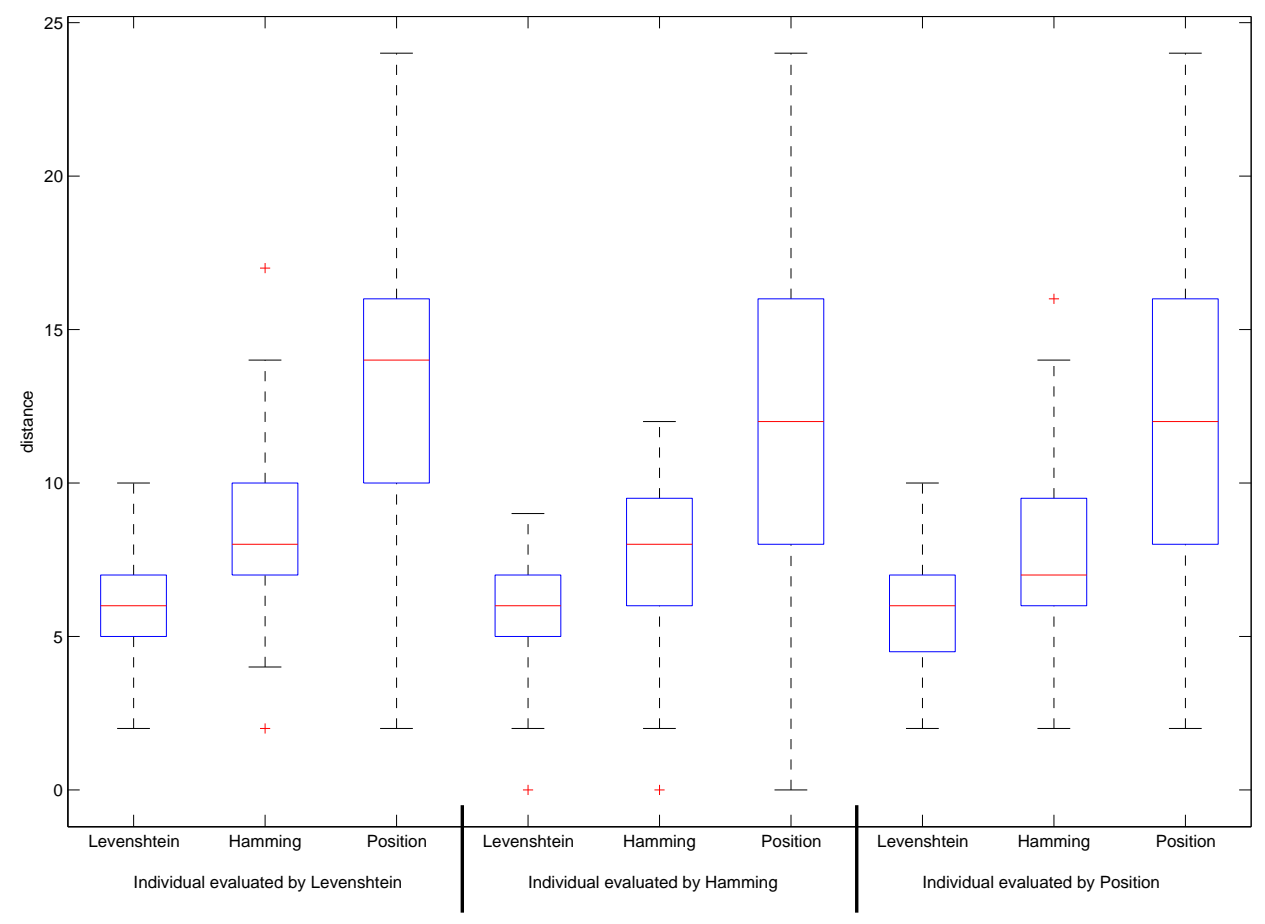

Fig. 4. Whisker-box plots for the test results on three sequence metrics from all three best individuals obtained from training.

[3] R. de Neufville and A. Odoni, Airport Systems: Planning, Design, and Management, ser. Aviation Week Book. Mcgraw-hill, 2002. [Online]. Available: http://books.google.com.au/books?id=9khd5ns3kz4C

[4] H. Balakrishnan and B. Chandran, "Scheduling aircraft landings under constrained position shifting," in AIAA guidance, navigation and control conference and exhibit, 2006.

[5] F. Neuman and H. Erzberger, Analysis of delay reducing and fuel saving sequencing and spacing algorithms for arrival traffic. Citeseer, 1991.

[6] C. D. Wickens, A. S. Mavor, and J. P. McGee, Flight to the future: Human factors in air traffic control. National Academies Press, 1997.

[7] W. Zhao, J. Tang, S. Alam, A. Bender, and H. A. Abbass, "Evolutionarycomputation based risk assessment of aircraft landing sequencing algorithms," in Distributed, Parallel and Biologically Inspired Systems. Springer, 2010, pp. 254-265.

[8] H. Abbass, A. Bender, S. Gaidow, and P. Whitbread, "Computational red teaming: Past, present and future," Computational Intelligence Magazine, IEEE, vol. 6, no. 1, pp. 30-42, 2011.

[9] M. O. Rabin, "Probabilistic automata," Information and control, vol. 6, no. 3, pp. 230-245, 1963.

[10] D. Goldberg, Genetic Algorithms in Search, Optimization, and Machine Learning, ser. Artificial Intelligence. Addison-Wesley, 1989. [Online]. Available: http://books.google.com.au/books?id=3_RQAAAAMAAJ

[11] S. Donikian, "Hpts: A behaviour modelling language for autonomous agents," in Proceedings of the Fifth International Conference on Autonomous Agents, ser. AGENTS '01. New York, NY, USA: ACM, 2001, pp. 401-408. [Online]. Available: http://doi.acm.org/10.1145/ 375735.376382

[12] T. M. Mitchell, "Machine learning. 1997," Burr Ridge, IL: McGraw Hill, vol. 45, 1997.

[13] E. Vidal, F. Thollard, C. De La Higuera, F. Casacuberta, and R. C. Carrasco, "Probabilistic finite-state machines-part i," Pattern Analysis and Machine Intelligence, IEEE Transactions on, vol. 27, no. 7, pp. 1013-1025, 2005.

[14] D. B. Fogel, "Evolving behaviors in the iterated prisoner's dilemma," Evolutionary Computation, vol. 1, no. 1, pp. 77-97, 1993.

[15] D. Fogel, "An introduction to simulated evolutionary optimization," Neural Networks, IEEE Transactions on, vol. 5, no. 1, pp. 3-14, 1994.
[16] H. A. Abbass, "Learning regularities and patterns using probabilistic finite state machines," in 7th Asia-Pacific Conference on Complex Systems, 2004.

[17] V. I. Levenshtein, "Binary codes capable of correcting deletions, insertions and reversals," in Soviet physics doklady, vol. 10, 1966, p. 707.

[18] A. M. Steane, "Error correcting codes in quantum theory," Physical Review Letters, vol. 77, no. 5, p. 793, 1996. 